\title{
Discours
}

Revue de linguistique, psycholinguistique et

informatique. A journal of linguistics, psycholinguistics and computational linguistics

$20 \mid 2017$

Varia

\section{Ou comme ça, machin et autres marqueurs d'indétermination dans les listes}

\section{Marie-José Béguelin et Gilles Corminboeuf}

Édition électronique
URL : http://journals.openedition.org/discours/9275

DOI : $10.4000 /$ discours. 9275

ISSN : 1963-1723

Éditeur :

Laboratoire LATTICE, Presses universitaires de Caen

\section{Référence électronique}

Marie-José Béguelin et Gilles Corminboeuf, « Ou comme ça, machin et autres marqueurs

d'indétermination dans les listes », Discours [En ligne], 20 | 2017, mis en ligne le 22 septembre 2017 consulté le 30 avril 2019. URL : http://journals.openedition.org/discours/9275;DOI : 10.4000/ discours. 9275 

Revue de linguistique, psycholinguistique et informatique

\title{
Ou comme ça, machin et autres marqueurs
} d'indétermination dans les listes

\author{
Marie-José Béguelin \\ Université de Neuchâtel - FNS nº 100012-146773 \\ Gilles Corminboeuf \\ Université de Fribourg - FNS n $100012-146773$
}

Marie-José Béguelin, Gilles Corminboeuf, «Ou comme ça, machin et autres marqueurs d'indétermination dans les listes», Discours [En ligne], 20 | 2017, mis en ligne le 22 septembre 2017.

URL: http://discours.revues.org/9275

Titre du numéro: Varia

Coordination: Shirley Carter-Thomas \& Laure Sarda 



\title{
Ou comme ça, machin et autres marqueurs d'indétermination dans les listes
}

\author{
Marie-José Béguelin \\ Université de Neuchâtel - FNS nº $100012-146773$ \\ Gilles Corminboeuf \\ Université de Fribourg - FNS nº 100012-146773
}

Cet article traite de deux marqueurs de fin d'énumération ou de liste que l'on rencontre dans le «Corpus oral de français de Suisse romande » (OFROM). II s'agit d'une part de la séquence ou comme ça, dont la fréquence caractérise semble-t-il le corpus en question, et d'autre part de machin, plus uniformément répandu en français parlé contemporain d'Europe. Les emplois ciblés par notre étude sont du type suivant:

[1] certaines se maquillent déjà ou bien mettent des robes ou euh + des ba- justement des chaussures un peu féminines comme des ballerines ou comme ça (unine11-csa)

[2] je lui ai donné un millier de conseils va voir un psy va voir euh prends-toi en main fais quelque chose euh machin (unineo8-vwa)

Sans nous limiter aux contextes énumératifs, nous examinons les conditions dans lesquelles ou comme ça et machin sont utilisés dans les données OFROM. Nous étudions leurs fonctionnements sémantiques respectifs, le type d'indétermination auquel ils sont associés, et la manière dont ils en viennent à assumer, en fin de liste, des rendements pragmatiques comparables. Au passage, nous mettons en évidence les contextes morphosyntaxiques qui favorisent, en français contemporain, une réinterprétation du substantif machin comme une particule d'extension, voire d'indétermination.

Mots clés: liste, énumération, discours rapporté, indétermination, approximation, particules d'extension, transcatégorisation, machin, ou commeça

This article deals with two extension particles (or "general extenders") found in the OFROM corpus (French spoken in Switzerland). On the one hand, we study the sequence ou comme ça ("or like that"), whose high frequency seems to be characteristic of the OFROM corpus. On the other hand, we study the noun machin ("thingy", "whatsit"), which is more uniformly spread in contemporary European spoken French. The examples studied are of the following types:

[1] certaines se maquillent déjà ou bien mettent des robes ou euh + des ba-justement des chaussures un peu féminines comme des ballerines ou comme ça (unine11-csa)

[2] je lui ai donné un millier de conseils va voir un psy va voir euh prends-toi en main fais quelque chose euh machin (unineo8-vwa)

The research is not limited to enumerative contexts. We examine all the uses of ou comme ça and machin in the OFROM data. We study their semantic functions, the type of vagueness they are associated with, and the way in which they come to assume comparable pragmatic functions at the end of the list. We highlight the morphosyntactic contexts that favor a reinterpretation of the name machin as an extension particle, or as a vagueness marker.

Keywords: list, enumeration, reported speech, indetermination, approximation, extenders, transcategorization, machin, ou comme ça 


\section{Introduction ${ }^{1}$}

\subsection{Objet de l'étude}

La présente étude porte sur ou comme ça et machin, en particulier lorsqu'ils fonctionnent comme «extenseurs», "particules d'extension» (Ferré, 2009; angl. «general extenders», Overstreet, 2005), ou encore «clôtureurs» (Kahane et Pietrandrea, 20I2), «prolongateurs» (Gadet, 20I7). En ce cas, ils signalent l'achèvement d'une énumération ou d'une liste, indiquant, de manière un peu paradoxale, que l'énumération reste ouverte et pourrait être prolongée. Voir les exemples [I] et [2]:

[I] certaines se maquillent déjà ou bien mettent des robes ou euh + des ba- justement des chaussures un peu féminines comme des ballerines ou comme ça

(unineII-csa $\left.{ }^{2}\right)$

[2] je lui ai donné un millier de conseils va voir un psy va voir euh prends-toi en main fais quelque chose euh machin

(unineo8-vwa)

En dépit de ce qui les différencie, les deux formules qui terminent [I] et [2] semblent plus ou moins interchangeables: elles ont pour point commun d'exprimer une forme d'indétermination. Nous nous proposons d'analyser leurs fonctionnements respectifs, tout en les comparant avec d'autres extenseurs dont la description est désormais bien établie dans la littérature: notamment et tout, (et) tout ça (Johnsen, 2OII et 20I7; Secova, 20I4 $4^{3}$.

Dans ce qui suit, nous recourrons à la notion de «liste» introduite par BlancheBenveniste et l'équipe du Groupe aixois de recherche en syntaxe (GARS) pour décrire les phénomènes de bribes, de reformulation et d'énumération. Blanche-Benveniste définit la liste comme un phénomène de coordination qui consiste à aligner (ou «entasser») sur l'axe syntagmatique «un ensemble de réalisations lexicales possibles pour un même élément régi, ces réalisations formant un paradigme». Ces réalisations entretiennent entre elles «une relation d'équivalence telle que l'on pourrait les représenter par une même pro-forme» (Blanche-Benveniste, 20II : I68). Ainsi, l'exemple [I] pourrait être synthétisé sous la forme «mettre quelque chose» (voir Willems, 1998: 140); de même, l'exemple [2] équivaut à «je lui ai donné un millier de conseils tels que ceux-ci». Quand elles concernent des places de rection, les listes «ne nous font pas passer d'une unité syntaxique à une autre; elles piétinent sur le même emplacement syntaxique» (Blanche-Benveniste, 1987, citée par Johnsen, 20II : 490). La notion de liste a aussi

1. Cette recherche s'inscrit dans le cadre du projet FNS IoooI2-I46773 «Marqueurs corrélatifs entre syntaxe et analyse du discours» dirigé par M.-J. Béguelin. Ont été, à un moment ou à un autre, des collaborateurs du projet: G. Corminboeuf, F. Delafontaine, F. Gachet, A. Guryev, L. A. Johnsen et P. Montchaud.

2. Sauf mention contraire, tous les exemples cités sont extraits de la banque de données OFROM, qui sera présentée plus bas (section I.2).

3. Cet article contient une revue des travaux sur les «general extenders», à laquelle nous nous permettons de renvoyer (Secova, 20I4: 282-284). 
été appliquée au-delà de la rection, pour décrire des énumérations de constructions verbales relevant du niveau discursif (Bilger, 1982; Johnsen, 20II). L'exemple [2] en fournit une illustration. Y sont «alignées» trois clauses à l'impératif qui forment une période-liste (Groupe de Fribourg, 20I2), ponctuées in fine par machin ${ }^{4}$. Les faits ciblés par cet article peuvent concerner les deux cas de figure de la liste, comme le montre une analyse «en grille» sommaire des exemples [I] et [2] présentée sous [3a] et [3b]:

[3a] mettent des robes ou euh + des ba-

justement des chaussures [...] comme des ballerines

ou comme ça

(cf. $§ 2.2$ pour une discussion)

[3b] va voir un psy

va voir euh

prends-toi en main

fais quelque chose euh

machin

Du point de vue fonctionnel, il est admis que la liste peut servir à spécifier progressivement un référent donné, à énumérer une série de référents, ou encore à corriger ou à réparer le dire, sans qu'il y ait toujours moyen de trancher absolument entre ces diverses interprétations.

\subsection{Données empiriques et méthodologie}

La présente recherche exploite une ressource en cours de développement à l'université de Neuchâtel: le corpus OFROM («Corpus oral de français de Suisse romande»), librement accessible en ligne (Avanzi et al., 2012-2015). Cette banque de données se montait à environ 850000 mots en avril 2017. Mais au moment où la présente étude a été réalisée (automne 2015), elle en comptait 615000, ce qui représentait 222 locuteurs et 64 heures de parole.

Comme Secova (2014) l'a observé en confrontant, dans une perspective de sociolinguistique variationniste, trois corpus oraux ${ }^{5}$, les extenseurs de liste sont très courants en français parlé informel. Les cinq plus fréquents sont, dans les données qu'elle a étudiées: tout ça, et cetera, et tout, (les/des) choses comme ça, et tout ça ${ }^{6}$, avec de fortes

4. On passe alors de la micro- à la macro-syntaxe. Il n’y a pas en ce cas de «piétinement» sur une position syntaxique, mais un parallélisme syntaxique et fonctionnel entre clauses successives.

5. Un corpus personnel dit «Corpus Secova» (II heures d'enregistrement de jeunes locuteurs parisiens), le "Corpus de français parlé parisien des années 2000» (CFPP2000 - environ 38 heures d'enregistrement), collectés tous deux entre 2006 et 2009 ; ils sont comparés à un corpus plus ancien, le «Beeching corpus», recueilli entre 1980 et 1990 dans différentes parties de la France (Secova, 20I4: 284-286).

6. Johnsen (2017: 223) cite bien d'autres formules encore, telles et ainsi de suite, et autres $N$, et compagnie, et consorts, et tout et tout, et tout le toutim, et tout le reste, et tout le tremblement/le tralala, etc., auxquelles on peut encore ajouter l'emprunt à l'italien e tutti quanti. 
disparités dans la sélection des marqueurs. Ainsi, le corpus personnel de Secova, exclusivement composé d'enregistrements de jeunes adultes vivant à Paris ou originaires de cette ville, privilégie quelques séquences d'ailleurs inégalement représentées : et tout (I5s exemplaires), tout ça (9), macbin (9), (ou) un truc comme ça (8), d'autres formules apparaissant de manière plus sporadique (voir Secova, 20I4: 303). Sensible à l'âge des locuteurs, la sélection des formules l'est également quant au facteur géographique. Le français du Québec connaît ainsi des extenseurs non représentés en français d'Europe: des affaires comme ça, toute l'affaire, des choses de même, des patentes comme ça/de même, tout le kit (voir Dubois, 1992, cité par Johnsen, 2017: 223 n.; Secova, 2014: 288). Dès lors, la question se pose de savoir si les données orales de Suisse romande présentent ou non des particularités dans l'emploi des extenseurs de liste.

Johnsen (20II, 20I7) s'est de son côté intéressée, dans une perspective de sémantique référentielle, aux occurrences de et tout et (et) tout ça dans les données OFROM et CFPP2000 (Branca-Rosoff et al., 2000). Les occurrences brutes, tous usages confondus, dont elle fait état, se montent à 307 (et) tout ça et 392 et tout dans OFROM et à 333 (et) tout ça et 194 et tout dans CFPP200o (recherche de février 20I7, dans Johnsen, 2017: $227 \mathrm{n}$.). Dans les deux corpus, l'emploi en fin de liste concerne, selon les comptages qu'elle a opérés, entre 80 et $85 \%$ des occurrences pour et tout et entre 52 et $56 \%$ pour tout ça (Johnsen, 20I7: 227) ${ }^{7}$. Dans l'attente d'une étude comparative plus poussée, on peut donc estimer que l'usage de et tout, tout ça, et tout ça ne diffère pas de manière essentielle dans les deux corpus, en dépit d'une présence apparemment plus forte de et tout dans le corpus suisse romand. Celui-ci contient cependant aussi, en nombre non négligeable, la formule ou comme ça- qui à notre connaissance n'a pas encore été décrite - ainsi que machin, dont l'emploi en tant qu'extenseur est signalé par Halmøy (2006) et par Secova (2014: 287), mais qui, sauf erreur ou omission de notre part, n'a pas encore donné lieu à une étude systématique dans un corpus de français parlé.

La lexie comme ça, non précédée de ou, connaît des emplois variés (voir, à ce sujet, Corminboeuf, 20I7; Béguelin, 20I6: III-II2). Dans l'un d'eux, elle fonctionne comme un marqueur d'approximation, sans être forcément liée au phénomène de liste. Pour les besoins de la présente étude, nous avons enquêté exclusivement sur les usages de comme ça précédé du coordonnant disjonctif ou, séquence qui révélait des affinités particulières avec le contexte énumératif et dont il était possible de traiter l'intégralité des occurrences dans le corpus de référence. Nous n'avons pas relevé en revanche - du moins de manière méthodique - les séquences développées du type ou quelque chose comme ça et ou des trucs/des choses comme ça ${ }^{8}$. Dans le cas

7. L'auteur avance ces chiffres avec toute la prudence requise. Elle donne des données chiffrées grosso modo concordantes, relatives à d'autres corpus, dans un article de $201 \mathrm{I}$ (Johnsen, 20II : 488 n. 4).

8. En voici deux exemples oraux issus d'OFROM et un exemple écrit tiré de Frantext:

[i] [...] un donut ou bien un un coca ou un fanta ou quelque chose comme ça

[ii] [...] des cours de dessin ou des trucs comme ça

[iii] Je crois qu'il s'appelle Jacquier, ou quelque chose comme ça.

(Frantext: B. Clavel, La maison des autres [1962], Paris, J'ai lu, 1993, p. 485) 
de machin, la situation se présentait plus simplement, et l'analyse a pu porter sans difficulté particulière sur la totalité des occurrences du lemme.

Sur la base des choix qui viennent d'être présentés, nous avons relevé dans OFROM, aux fins de l'étude:

- 78 occurrences de ou comme ça (dont 64 en clôture de liste),

- 97 occurrences de machin (dont une trentaine en tant que particule d'extension ou d'approximation).

Nous procéderons à une analyse détaillée des deux séries, sans nous limiter aux seuls contextes énumératifs. La prise en compte de la totalité des emplois apporte en effet, comme on le verra, un éclairage bienvenu sur l'emploi qui est au cœur de ce travail, celui d'extenseur de liste. La perspective adoptée, attentive aux facteurs morphosyntaxiques, nous conduira à contester les explications de l'évolution de nos marqueurs qui s'appuient sur un scénario préétabli de grammaticalisation, fondée sur l'idée (à nos yeux naïve) d'une soi-disant "perte» de contenu lexical et d'une décatégorisation ${ }^{9}$.

\subsection{Aperçu des questions ouvertes}

11 Dans cette étude, nous traiterons en priorité des modalités d'interprétation de ou comme ça et de macbin, laissant provisoirement de côté un certain nombre de problèmes dont chacun mériterait une étude en soi. Parmi ceux-ci, on peut citer:

- la distinction entre listes additives vs substitutives (voir Overstreet, 2005), i. e. entre les listes refermées par des extenseurs introduits par et (et cetera, et tout, et compagnie...) ou au contraire par ou (ou comme ça, ou je sais pas (quoi/comment), ou j'en sais rien, ou n'importe, ou quoi que ce soit...). Pourquoi, en parallèle à ou comme ça et et tout, ne trouve-t-on que rarement les suites et comme ça et ou tout ça ${ }^{10}$ ?

- la question d'une éventuelle variation linguistique (voir la section I.2). Des pointages sur la représentation de la forme ou comme ça dans d'autres corpus de français parlé donnent des résultats contrastés. Alors que la forme est fréquente dans OFROM (78 exemples pour 615000 mots), on n'en trouve

9. Un tel scénario diachronique est convoqué dans le cas de machin par Secova (2014:287 et 290): pour une critique, voir Johnsen (2017: 226). Il l'est aussi, quoique différemment, par Mihatsch (2006), qui voit dans machin non un extenseur mais une particule d'hésitation. Ces hypothèses seront reprises et discutées à la section 3.4.

10. Dans le corpus de référence, nous n'avons relevé qu'un seul exemple avec et comme ça en position d'extenseur. Voici l'extrait:

[iv] L1: ben voilà ça j'ai été témoin souvent +

L2: ah [oui]

L1: [euh de $\mathrm{m}-$ ] ouais de mépris et comme ça

Notre corpus de référence ne fournit aucune occurrence de ou tout ça et ou tout en contexte énumératif; Johnsen (2017: 224) en mentionne toutefois un exemple trouvé dans le corpus «Phonologie du français contemporain» (PFC). 
dans CFPP2000 (version automne 2015) qu'une demi-douzaine d'exemples pour 535000 mots, dont celui-ci:

[4] par rapport à d'autres quartiers de Paris + tu dirais que + tu m'as parlé de quartiers plus riches ou comme ça $(\mathrm{mm})$ est-ce que y aurait aussi des différences dans le parler des gens ou pas à ton avis

(oral, CFPP2000, 13-I)

Par ailleurs, le corpus récent «Français parisien multiculturel» (MPF 850000 mots; voir Gadet, 2017) ne livre qu'un seul exemple de ou comme ça, qui semble, à première vue au moins, étranger à l'emploi d'extenseur qui nous intéresse ici («ah mince ça s'écrit comme ça ou comme ça») ${ }^{11}$. L'emploi de machin comme prolongateur est au contraire présent dans les trois corpus mentionnés.

Dans les écrits destinés à la publication, ou comme ça semble rarissime, comme le montre la difficulté qu'il y a à attester ne serait-ce qu'un exemple dans Frantext ${ }^{12}$ :

[5] Je ne suis pas européaniste, ou matérialiste, ou comme ça.

(Frantext: A. Malraux, Antimémoires [1967], Paris, Gallimard, 1996, p. 446)

L'emploi de machin comme particule d'extension est lui aussi difficile à attester à l'écrit, bien qu'il ait vocation à paraittre dans des passages mimant l'oral, comme semble l'indiquer l'exemple [5o] infra.

Toutes grossières qu'elles sont, ces quelques observations invitent à se demander s'il n'y a pas, à propos de ou comme ça, un fait de variation diatopique: la différence constatée dans la fréquence d'utilisation serait-elle liée à un particularisme régional (voir ci-dessus la comparaison chiffrée des occurrences dans OFROM, CFPP2ooo et MPF) ? Ou faut-il y voir plutôt, du fait que les extenseurs de liste se renouvellent rapidement, un effet de l'«âge» du corpus, OFROM étant plus récent que CFPP2000? Jusqu'à plus ample informé, les résultats relatifs au corpus MPF orientent vers la première hypothèse.

\subsection{Structure de l'étude}

La section 2 aura pour objet les usages de ou comme ça dans la base OFROM. L'approche, de type distributionnel, visera à identifier les contextes syntaxiques dans lesquels apparait cette suite et les rendements auxquels elle est associée. Puis nous passerons à l'examen de machin (section 3), dont les occurrences dans OFROM seront relevées et analysées tous usages confondus, en vue de mieux comprendre comment l'emploi en tant que prolongateur s'articule avec l'emploi nominal classique. En guise de conclusion (point 4) nous confronterons les comportements de ou comme

11. Nous remercions F. Gadet et P. Cappeau de nous avoir aimablement transmis cette information.

12. Hormis l'exemple [5], la base Frantext contient huit occurrences de ou comme ça, dont six qui apparaissent dans la lexie comme ci ou comme ça. Restent deux exemples où la séquence n'a pas non plus le statut d'extenseur. 
ça et de machin qui, en dépit de leurs différences aux plans morphosyntaxique et sémantique, assument à l'occasion des fonctions pragmatiques similaires.

\section{Usages de ou comme ça dans la base OFROM}

Nous avons identifié cinq contextes d'apparition de ou comme ça dans notre corpus, que nous détaillons en 2.2 , après avoir proposé une analyse sémantique de la séquence (section 2.I). Nous verrons que deux des cinq contextes relèvent en propre du phénomène d'énumération. En 2.3, nous résumons sous la forme d'un tableau les fonctions pragmatiques repérées et les liens qu'elles entretiennent.

\subsection{Analyse sémantique de la séquence ou comme ça}

Au plan compositionnel, ou comme ça se laisse trivialement découper en trois constituants: le coordonnant ou, le comparant comme et le démonstratif ça. Au regard des séquences ou quelque chose/un truc/des choses comme ça, également attestées, la formule ou comme ça peut être considérée comme elliptique.

- Le ou est disjonctif, au contraire du et additif de et tout, et tout ça. Ce ou a ici une valeur inclusive, comme dans libellule ou demoiselle ${ }^{13}$, ou dans cet exemple cité par la Grammaire méthodique du français: «Des passeports seront délivrés aux ressortissants du pays ou aux personnes ayant épousé un ressortissant du pays» (Riegel et al., 2009: 88I). Autrement dit, il ne s'agit pas d'un ou exclusif ${ }^{14}$ comme dans les couples vaincre ou mourir, fromage ou dessert.

- Le pronom ça à référence vague, dépourvu de trait de catégorisation (Cadiot, 1988; Maillard, 1989; Corblin, 1995; Sales, 2008; Johnsen, 2017), anaphorise, quoique de manière floue, l'information récemment mise en mémoire.

- Le comme, «instrument didentification» (Fuchs et Le Goffic, 2005), introduit une comparaison: ou comme ça indique que d'autres référents pertinents (désignés par l'élément zéro) pourraient être mentionnés, en relation d'analogie au moins présumée avec le ou les référents désignés par ça. Est ainsi invoquée une classe aux contours flous de référents alternatifs possibles - que cette classe soit préconstruite ou éventuellement ad hoc.

L'ellipse d'un syntagme nominal (SN) et la présence du pronom ça sont donc au principe de l'indétermination constitutive de la formule ou comme ça prise dans son ensemble.

13. Le Trésor de la langue française informatisé (TLFi) traite libellule et demoiselle comme des synonymes.

14. À noter que le TLFi définit ou comme une «conjonction de coordination disjonctive, indiquant une alternative qui a valeur de distinction pouvant aller jusqu'à l'exclusion»; Riegel et al. (2009) assimilent pour leur part alternatifà exclusif. Dans ce qui suit, nous nous en tiendrons à l'usage des termes alternatif, alternative qui figurent dans le TLFi. 
Qu'est-ce qui distingue dès lors ou comme ça de et tout, (et) tout ça? D'après Johnsen (2017: 22I), tout ça extenseur de liste «cumule une fonction résomptive et "extensible"». En effet, «il comprend dans sa référence celle des items (de l'item) préalablement mentionné(s)», tout en suggérant que «la liste entreprise ne reflète pas exhaustivement sa référence» (Johnsen, 2017: 237). Autrement dit, le syntagme tout ça peut désigner, en fin de liste, un ensemble «dont la teneur est partiellement implicite au niveau référentiel», et dont l'interlocuteur est invité à «inférer d'autres membres potentiels» (Johnsen, 2017: 237). Dans ou comme ça, exempt de tout, on ne décèle pas la même ambition totalisante. Il y a, en revanche, ouverture sur des référents alternatifs potentiels qui, en raison de la relation d'analogie instaurée par comme, sont présentés comme étant en rapport avec le ou les référent(s) mentionné(s) au préalable et pointé(s) par ça.

En résumé, ou comme ça joue sur l'évocation vague de référents alternatifs en relation d'analogie avec ce qui vient d'être mentionné, alors que et tout, (et) tout ça réalisent une totalisation portant sur un ensemble flou, que l'interlocuteur est invité à enrichir par voie inférentielle (Bilger, 1989; Johnsen, 20II et 2017). Face à la prétention totalisante de et tout, (et) tout ça, la formule ou comme ça apparaît comme moins rigidifiante, en raison de la présence du ou disjonctif.

\subsection{Les cinq contextes identifiés}

Comme il sera fait plus bas pour machin (section 3), nous établissons sur une base distributionnelle les conditions d'apparition de ou comme ça, qu'il s'agisse de contextes énumératifs ou non énumératifs. Parmi les 78 occurrences du corpus, il nous semble possible, en fonction de la position occupée par ou comme ça, de distinguer les cas de figure suivants.

\subsubsection{Type 1: après une indication chiffrée}

Dans ce premier type, l'extenseur suit la mention d'un chiffre, typiquement au moyen d'un numéral cardinal:

[6] $\mathrm{LI}$ : ben oui mais c'est pour ça que j'ai fait $\mathrm{l}^{\prime} \mathrm{AG}{ }^{15}$ parce j'avais regardé ben combien c'était par mois

L2: $\mathrm{mmh} \mathrm{mmh}$

Lr: juste l'abonnement de parcours jusqu'à Brigue $+{ }^{16}$ pis c'était je crois deux cent vingt-cinq ou comme ça pis l'abonnement général c'était deux cent quarante

(unineII-csa)

[7] leur fille aînée qui a + je sais pas quel âge elle a en fait enfin elle a une trentaine d'années ou comme ça

(unineo8-yba)

15. AG = l'abonnement général pour le réseau ferroviaire suisse.

16. Les «+» marquent des pauses. 
Le locuteur «se couvre» en prévenant, de la part de l'interlocuteur, une inférence du type: «celui qui parle est certain de la précision du chiffre». Ainsi, dans l'exemple [6], donner un chiffre très précis pourrait porter à croire que le locuteur connait le montant avec certitude. L'utilisation de ou comme ça, associée à la modalisation je crois, lui permet d'adopter une posture moins tranchée - et de se ménager une possibilité de repli.

Dans l'exemple [7], la locutrice confesse de prime abord son ignorance (je sais pas quel âge elle a), semble ensuite se raviser (elle a en fait), puis elle fournit une indication approximative (une trentaine d'années), avant de clore en ouvrant une alternative (ou comme ça); la formule choisie suggère cependant qu'elle ne peut tomber bien loin de l'indication précédemment avancée. Les locuteurs réalisent volontiers une telle redondance d'indications vagues. Reste qu'une alternative est suggérée, comme s'il $y$ avait une autre solution (chiffrée) possible ${ }^{17}$.

\subsubsection{Type 2 : après les premiers éléments d'une énumération à plusieurs items}

Avec le type 2, nous entrons dans la catégorie des contextes qui peuvent être décrits comme énumératifs. Il s'agit du premier des deux contextes de ce genre que nous avons repérés. La séquence ou comme ça se trouve alors placée après les premiers items d'une énumération, en fonction d'extenseur:

[8] LI : d'autres sports euh non + je suis un peu un touche-à-tout donc si on me propose d'aller faire un sport j'ai eu été jouer au volley avec des copains au badminton-

$\mathrm{L} 2: \mathrm{mmh}$

LI : au:: ++ squash ou comme ça mais c'est pas euh je pratique pas ces sports quoi je vais comme ça euh pour rigoler

(unineII-fdb)

[9] j'avais décidé de faire euh quelque chose de peut-être humanitaire + d'aller dans un orphelinat ou comme ça

(unineo8-yba)

[ı] certaines se maquillent déjà ou bien mettent des robes ou euh + des ba- justement

$=[\mathrm{I}]$ des chaussures un peu féminines comme des ballerines ou comme ça (unineII-csa)

Dans l'exemple [8], l'énumération, qui comporte trois items (au volley, au badminton, au squash), justifie le choix du composé touche-à-tout pour caractériser la façon dont le protagoniste s'adonne à la pratique sportive.

Dans l'exemple [9], l'indice prégnant est l'intonation continuative sur bumanitaire, qui projette une suite. Pour cette occurrence, deux interprétations sont possibles,

17. Dans ce type I, l'indice récurrent est la présence d'un numéral cardinal, comme dans [6]. Mais ce n'est nullement une condition nécessaire, comme le montre le substantif trentaine dans [7], qui nomme un ensemble flou (d'environ trente éléments). 
en fonction de la portée du marqueur ou comme ça: a-t-il pour opérande un orphelinat? Autrement dit, complète-t-il le second terme? Ou alors - seconde lecture - constitue-t-il le troisième terme de la liste, ouvrant ainsi sur une autre activité? Ni les indices contextuels, ni la prosodie de la séquence ne permettent de trancher.

Dans l'exemple [Io], les ou (bien) orientent vers l'hypothèse d'une portée globale de ou comme ça, avec l'interprétation: «certaines se maquillent, ou mettent des robes, ou des chaussures féminines [ou portent des bijoux]»; l'extenseur suggère qu'il existe une suite possible à l'énumération des actions jugées typiquement féminines. Au contraire, le premier comme pourrait orienter vers une portée locale de l'extenseur, avec la lecture: "certaines mettent des chaussures un peu féminines comme des ballerines [ou des bottines, etc.]»; ou comme ça évoquerait alors de manière vague des référents alternatifs, relevant de la classe des types de chaussures féminines. Dans cette seconde interprétation, l'exemple [ı] refléterait le type 3 (voir infra). Plusieurs exemples du corpus présentent de telles ambiguïtés. Nous y reviendrons en 2.3.

\subsubsection{Type 3: après un élément ouvrant une énumération réduite à un seul item}

Le marqueur ou comme ça apparaît très communément précédé d'un seul item. Suivant Johnsen (20II) et Secova (20I4), nous analysons ces occurrences comme relevant en propre du phénomène de liste:

[II] ben comme je disais on sortait souvent le soir boire un petit verre ou comme ça + et y a pas un endroit où y pas un musicien et qui fait + qui chante et tout et pis qui + qui met l'ambiance vraiment

(unineo8-yba)

[I2] parce que maintenant toutes ces voisins-là c'est des dames qui sont seules + qui ont supprimé leur jardin alors euh + je je leur donne une tête de salade ou comme ça (unineII-jsa)

[13] et puis à New York on a trouvé un appartement pour euh deux cents francs euh + pour une semaine ++ pour cha- pour + ben chacune hein deux cents francs mais + tu vois comparé à un hôtel ou comme ça c'est ++ puis c'est un appartement quoi c'est ++++ donc ça c'est vraiment chouette

(uninerI-tpa)

[14] LI: [...] c'est quand même + plus facilement la langue maternelle qui est apprise aux enfants

L2: $\mathrm{mmh} m \mathrm{mh}$ + ouais ben c'est sûr [que-]

Lr: [c'est] normal + ouais les les gens qui ont épousé une + je sais pas une Espagnole ou comme ça la maman elle parle plus facilement espagnol avec ses enfants + que le papa

(uniner5-029) 
Les exemples [II] à [I4] présentent le même schéma: la mention d'un prototype de la catégorie évoquée, suivi de la particule d'extension. Ainsi boire un petit verre est l'archétype de ce que font les jeunes quand ils sortent en soirée - en plus de discuter, de se retrouver entre amis, d'écouter de la musique, etc. On notera également ici la présence de l'extenseur et tout qui, du moins dans ce contexte, joue un rôle analogue à celui d'ou comme ça ${ }^{18}$. Dans l'exemple [I2], une tête de salade désigne de même le prototype de ce que l'on plante dans un jardin potager. L'extenseur fournit un recours commode, afin de s'éviter une énumération. Idem pour [I3], où un hôtel constitue le cas banal des solutions d'hébergement à New York.

L'extrait [14] présente le même cas de figure, avec maman espagnole comme exemplaire typique des familles bilingues. Mais l'extrait a aussi à voir avec les modalités du dire (je sais pas, comme dans [7] supra), dont il sera question à la prochaine section.

La configuration $\{\mathrm{SN}$ nommant un objet prototypique + ou comme ça\} constitue un procédé économique, dans la mesure où un seul item permet d'ouvrir, quoique de manière vague, sur tout un paradigme. Le prototype de la catégorie désignée par le SN suivi de l'extenseur «vaut» en quelque sorte pour l'intégralité de cette catégorie ${ }^{19}$.

Dans les faits présentés aux sections 2.2.2 et 2.2.3, ou comme ça produit clairement un effet de mitigation et de connivence entre interlocuteurs ${ }^{20}$, sur le mode du «on se comprend», «je vous épargne l'ensemble de la liste».

\subsubsection{Type 4 : «non-coïncidences du dire » (Authier-Revuz, 1995)}

La séquence ou comme ça peut apparaître dans un contexte qui présente des disfluences diverses. Voyons les extraits [I5] et [I6] :

[I5] non c'était plutôt euh + ouais des des discours genre peut-être comme dans le train ou comme ça en ayant fait une connaissance ou + dans des lieux publics je dirais voilà

(uniner5-023)

[16] c'est comme d'ailleurs tous les grands peintres Picasso euh + Braque ils ont ils ont tous senti + les choses même s'ils ont fait des peintures euh ++++ euh + surréalistes ou comme ça mais au fond ils sentaient comment était fait [...] (unineo8-jta)

18. Voir également l'exemple [I8] infra et cet extrait:

[v] on est beaucoup mieux équipé installé pour euh pour les soins pour les examens et pis tout + s'il y a des radios à faire ou comme ça

19. Pour ce cas où il n'y a qu'un seul désignateur suivi de ou comme ça, il arrive que l'élément ne dénote pas un objet, mais une situation ou un événement:

[vi] [...] parler à un prof de l'uni par exemple + j'essaierais de + de faire attention un peu à mes mots ou comme ça tu vois

20. Cet effet de connivence a été constaté depuis longtemps: Secova (2014: 288-289) mentionne notamment Dines (1980), qui a observé qu'il n'y avait jamais, de la part de l'interlocuteur, de demande d'éclaircissement après les extenseurs, mais plutôt un feedback de type approbatif. 
Dans l'exemple [i $]$, il est question des discussions que l'on peut avoir hors de la sphère privée, par exemple avec un(e) inconnu(e) et en présence d'autres personnes. On notera le rôle des marques d'hésitation (eub, des des) et l'accumulation des marques modales ou d'approximation comme genre, peut-être et je dirais qui laissent penser que la locutrice recherche une épithète ou une expansion quelconque apte à désigner un certain genre de discours; on voit bien la difficulté qu'il y a à trouver la formulation la plus adéquate. Le substitut insatisfaisant qu'elle trouve (des discours comme dans le train) ébauche une liste des situations où l'on observe ce type de discours. Un allongement syllabique sur train suggère d'ailleurs l'amorce d'une liste ${ }^{21}$.

Dans l'extrait [16], l'épithète surréaliste est actualisée faute de mieux (voir les pauses et les eub), et n'est sans doute pas perçue comme totalement satisfaisante par le locuteur (Braque n'est d'ailleurs pas catalogué, en général, comme un peintre surréaliste). L'ajout de ou comme ça est une façon de se prémunir contre une éventuelle objection et de se ménager, si l'on peut dire, une porte de sortie: la possibilité d'une rectification ultérieure est prévue d'avance ${ }^{22}$, la locutrice admet que son dire pourrait être différent, plus précis. Certains exemples témoignent ainsi d'un «à dire» «en souffrance», d'une "défaillance de l'énonciateur» (Authier-Revuz, 1995, t. $2: 639-640)$.

En produisant une énumération de plusieurs items (type 2) ou d'un seul item suivi d'un extenseur (type 3), le locuteur vise-t-il des référents distincts? Ou procède-t-il, comme dans le type 4, à une désignation approchée d'un seul et même référent, au moyen de tentatives successives pour le circonscrire (Blanche-Benveniste, 20II; Johnsen, 20II et 20I7) ? Voici la façon dont Johnsen, à propos de l'extenseur tout ça, présente la différence entre les deux procédés, en commentant l'extrait [I7] :

[17] nous on écoutait pas on écoutait pas peut-être que d'autres personnes des militaires tout ça qui étaient en France euh + suivaient mais d'ailleurs ces ces messages étaient des fois + abrégés et on comprenait pas ce que ça voulait dire

(oral, corpus «Choix de textes en français parlé» [CTFP]; cité par Johnsen, 2oır : 492)

En effet, dans cet exemple, on peut se demander si les classes des autres personnes et des militaires sont en relation de disjonction, auquel cas on aurait une addition des deux classes mentionnées, ou si elles entretiennent une relation inclusive: dans ce cas-là, on pourrait encore hésiter sur la teneur de la relation : la classe des militaires viendrait-elle spécifier, exemplifier ou reformuler celle des autres personnes? L'emploi

21. Dans cet extrait, la liste se poursuit au-delà de l'extenseur ou comme ça (en ayant fait une connaissance ou dans des lieux publics), ce qui signale que la locutrice ne se satisfait pas de la formulation initiale. La comparaison des fonctions respectives de ou comme ça et de voilà est révélatrice: avec ou comme ça, la locutrice ouvre un paradigme (tout en instillant un soupçon sur la formulation), alors qu'avec voilà (en fin d'extrait) elle entérine l'hyperonyme lieux publics.

22. On aurait sans doute avantage à distinguer ce rôle de qualification alternative de la fonction d'extenseur que remplit ou comme ça dans les types 2 et 3. 
de tout ça par la suite ne permet pas non plus d'identifier le type de relation en question, du fait qu'il semble compatible avec la saisie d'une totalité à valeur aussi bien additive qu'inclusive (englobante).

(Johnsen, 2011 : 492)

Tout comme [I7], les exemples [I5] et [I6] neutralisent cette opposition entre addition et inclusion.

\subsubsection{Type 5 : en clôture d'un segment de discours direct}

Ce dernier type est illustré par l'exemple [I8]:

[I8] la chose importante c'est vraiment d'avertir les gens tout de suite + de toutes les possibilités et pis ben + de ce que ça va leur coûter et pis tout et pis après ils ont aucun regret de dire ben + ah vous auriez dû me dire que je pouvais faire ça ou comme ça quoi ${ }^{23}$

(unineo8-fa)

L'extenseur ou comme ça borne ici la fin d'un discours direct. L'extenseur, bien qu'extérieur au discours rapporté, est néanmoins prononcé sans pause, comme s'il en faisait partie. La responsabilité du marqueur en gras incombe à l'énonciateur du discours citant, qui signale ainsi que le propos est rapporté en substance, de manière approximative, avec des options possibles.

Ce cas de figure est marginal dans notre corpus (un seul exemple), alors que machin, on le verra, est bien plus fréquent dans ce contexte.

\subsection{Fonctions pragmatiques}

La répartition des emplois de ou comme ça dans OFROM est résumée dans le tableau I. Les contextes énumératifs qui nous intéressent plus particulièrement sont représentés par les types 2 et 3 .

Il n'y a pas de transitions discrètes, mais une continuité entre les types d'emplois. Nous avons mentionné à dessein (supra, section 2.2) plusieurs exemples qui pouvaient être analysés de façons différentes. Ainsi, le placement d'un extrait dans le type 2 ou le type 3 dépend parfois de l'idée que l'on se fait de la portée de ou comme ça (à quel segment se rattache-t-il ?); voir exemple [9] supra. Et il a été question également des ambiguïtés entre les contextes énumératifs et les cas de changement de désignation (type 4); voir exemple [is] supra. Les contextes ambigus contribuent à l'effet de flou et d'indétermination produit par ou comme ça. Par ailleurs, les types I et 4 sont, à certains égards, assez proches des types 2 et 3 qui relèvent purement de l'énumération.

23. Les propriétés intonatives de cet exemple excluent une lecture du type «que je pouvais faire ça ou <faire> comme ça». 


\begin{tabular}{|c|c|c|c|}
\hline Type d'emploi & Exemple & $\begin{array}{l}\text { Aspect fonctionnel } \\
\text { remarquable }\end{array}$ & $\begin{array}{l}\text { Nombre } \\
\text { d'occurrences }\end{array}$ \\
\hline Type I & $\begin{array}{l}\text { si on doit attendre un } \\
\text { quart d'heure ou comme } \\
\text { ça on peut se permettre } \\
\text { d'en faire un deuxième } \\
\text { entre-temps }\end{array}$ & $\begin{array}{l}\text { Remise en question de } \\
\text { la précision du chiffrage } \\
\text { (ou redondance sur une } \\
\text { approximation). }\end{array}$ & 5 \\
\hline Type 2 & $\begin{array}{l}\text { on a juste le bruit des } \\
\text { feuilles du vent ou de la } \\
\text { pluie ou comme ça }\end{array}$ & \multirow{3}{*}{$\begin{array}{l}\text { Ouverture paradigma- } \\
\text { tique, place laissée aux } \\
\text { conjectures de l'auditeur: } \\
\text { stratégie de couverture } \\
\text { (bedging), effet de discours } \\
\text { moins catégorique. }\end{array}$} & 7 \\
\hline Ambigu $2 / 3^{24}$ & $\begin{array}{l}\text { quelque chose de peut- } \\
\text { être humanitaire }+ \text { d'aller } \\
\text { dans un orphelinat ou } \\
\text { comme ça }\end{array}$ & & 3 \\
\hline Type 3 & $\begin{array}{l}\text { je leur donne une tête de } \\
\text { salade ou comme ça }\end{array}$ & & 54 \\
\hline $\begin{array}{l}\text { Ambigu } \\
2-3 / 4\end{array}$ & $\begin{array}{l}\text { ouais des des discours } \\
\text { genre peut-être comme } \\
\text { dans le train ou comme ça }\end{array}$ & $\begin{array}{l}\text { Ouverture paradigmatique } \\
\text { ou soupçon sur l'appro- } \\
\text { priété de la dénomination. }\end{array}$ & 5 \\
\hline Type 4 & $\begin{array}{l}\text { des peintures euh ++++ } \\
\text { euh }+ \text { surréalistes ou } \\
\text { comme ça }\end{array}$ & $\begin{array}{l}\text { Soupçon sur l'appropriété } \\
\text { de la dénomination. }\end{array}$ & 3 \\
\hline Type 5 & $\begin{array}{l}\text { ils ont aucun regret de } \\
\text { dire ben }+ \text { ah vous auriez } \\
\text { dû me dire que je pouvais } \\
\text { faire ça ou comme ça quoi }\end{array}$ & $\begin{array}{l}\text { Discours direct rapporté } \\
\text { en substance. }\end{array}$ & $\mathbf{I}$ \\
\hline Total & & & 78 \\
\hline
\end{tabular}

Tableau 1 - Répartition des emplois de ou comme ça dans OFROM

Le résultat le plus remarquable qui ressort de ces indications chiffrées est la représentation élevée du type 3 (69,23\% des occurrences!). Dans ce contexte énumératif, la présence de l'extenseur ou comme ça peut s'expliquer par un facteur d'économie. Il serait en effet fastidieux de parcourir tout ou partie d'un paradigme, dans la mesure où un surcroît d'information n'apporterait pas d'effets cognitifs

24. Nous avons repéré trois exemples où il est difficile de décider entre les types 2 et 3 : ce sont des cas où la portée de ou comme ça demeure indécise.

25. Le sous-corpus compte deux exemples où on peut hésiter entre le type 3 et le type 4 et trois exemples où on peut hésiter entre le type 2 et le type 4 . La majorité de ces cas ambigus concerne donc la différence entre changement de désignation (type 4) et contexte énumératif (types 2 et 3 ). 
supplémentaires (Sperber et Wilson, 1989). La stratégie permet ainsi de réduire les coûts de formulation (côté locuteur) et de traitement (côté destinataire).

Si l'on adopte ce point de vue, les structures avec l'extenseur n'ont pas la même signification que des énumérations explicites; cela revient à dire que l'extenseur ne constitue pas un substitut à proprement parler d'items qui seraient sous-entendus et que l'allocutaire serait invité à reconstituer par inférence. La stratégie économique observée a en effet sa propre pertinence, l'allocutaire s'accommodant pleinement de cette indétermination.

Deux rendements principaux peuvent être dégagés. Énoncer «X, ou comme ça» remet en question, ou du moins laisse dans le flou:

I. tantôt l'adéquation du dire - que ce soit l'exactitude d'un chiffre X (type I), la justesse d'une dénomination $\mathrm{X}$ (type 4 ) ou la fidélité des paroles $\mathrm{X}$ rapportées au style direct (type 5$)^{26}$,

2. tantôt la complétude de $\mathrm{X}$, sa clôture (les contextes énumératifs: types 2 et 3).

D'une occurrence à l'autre, seul change le type d'item sur lequel porte ou comme ça. On pourrait généraliser en parlant d'un rendement de couverture (hedging), au service d'un discours qui se veut conciliant, non péremptoire, ouvert à d'autres façons de dire ${ }^{27}$. Ce rendement se manifeste de diverses manières: ouverture paradigmatique le plus souvent, mais aussi approximation dans le chiffrage, doute exprimé à propos de l'adéquation d'une dénomination, ou de la précision des paroles rapportées. Ainsi, il y a ou bien (i) saisie non péremptoire d'un objet discret $\mathrm{X}$, que cet objet prenne la forme d'un nombre (type I), d'une dénomination (type 4) ou d'un discours direct (type 5); ou bien (ii) saisie non péremptoire d'une classe $\mathrm{X}$, que cette classe soit un ensemble non clos (type 2), ou un ensemble flou dont tous les membres, sauf un, sont implicites (type 3).

En résumé, ou comme ça a pour fonction première d'«ouvrir»ou de dilater un référent mentionné au préalable. En cela, il est l'auxiliaire d'une forme de floutage, d'irrésolution; anticipant sur une objection possible, il remplit une fonction d'argumentation préventive et rend moins catégorique le discours qui le contient. Grâce à la présence du ou, cette fonction est explicitement assumée, alors qu'avec (et) tout ça un éventuel élargissement référentiel dépend d'une inférence de la part de l'interlocuteur (section 2.I). Cependant, si le locuteur laisse entendre avec ou comme ça que son dire pourrait être différent, plus précis, plus complet, plus abouti, etc., il suggère aussi que si on le complétait, on resterait dans le même ordre de référents (c'est le rôle supporté par comme ça).

26. Dans les types 4 et 5 , peut-être pourrait-on parler d'une portée méta-énonciative du ou comme ça.

27. «General extenders often function as bedges serving to mitigate or weaken the strength or directness of the utterance they punctuate, or add another possibility to the one ware raised» (Secova, 20I4: 289). 


\section{Le lexème postiche machin et ses fonctions en français parlé}

Cette section de l'article est consacrée au lexème du registre familier machin, dont on trouve, dans les corpus de français parlé contemporain, de fréquents emplois en tant que «prolongateur» ou particule d'extension (Halmøy, 2006; Secova, 20I4; Gadet, 2017). Dans ce rôle, machin entre en concurrence, et parfois aussi en association libre, avec les formules tous les trucs, (et) tout ça, et tout, et tout et tout, et pis tout, etc. :

[19] euh lui c'est vraiment un gars euh piano euh vin rouge + comme je dirais euh pièces de théâtre euh machin et tout

(oral, OFROM, unineo8-mba)

[20] mais tout ce qui est vie culturelle du quartier événements machin et tout ça ça me passe un peu au-dessus de la tête

(oral, CFPP200o)

[2I] elle était assez pauvre à l'époque et elle disait «oh j’aimerais bien que mes enfants aillent dans ce lycée-là plus tard il a l'air beau» et tout machin

(oral, CFPP200o)

Dans les cas où il joue le rôle de particule d'extension, machin apparaît dépourvu de déterminant; et spontanément, les transcripteurs le traitent comme invariable en nombre (voir l'exemple [20], où une version machins ne serait pas exclue a priori).

À la différence des prolongateurs concurrents, machin n'inclut pas d'élément à caractère résomptif (tel que ça), ni d'élément indiquant une totalisation (tout)... Quelles sont les incidences de ce statut non compositionnel? Et quels rapports les emplois de machin comme particule d'extension entretiennent-ils avec ses emplois nominaux classiques? L'analyse des 97 occurrences du lemme macbin dans la base OFROM nous fournira quelques indications utiles à ce propos ${ }^{28}$.

Pour commencer, nous rappellerons brièvement l'histoire du terme machin et les propriétés sémantiques qui lui sont reconnues (section 3.I). Ensuite, nous analyserons ses conditions d'apparition, en envisageant toutes ses occurrences. Sur des bases distributionnelles, nous dégagerons six catégories d'emploi ${ }^{29}$ qui seront commentées et illustrées en 3.2, puis présentées sous la forme d'un tableau récapitulatif, indiquant les rendements pragmatiques associés aux divers types (section 3.3). La partie se conclura par une réflexion sur le double statut catégoriel de machin en français parlé, substantif d'une part et particule d'extension de l'autre (section 3.4). Nous y mettrons en évidence les contextes syntaxiques qui, selon le corpus observé, ont pu permettre une réinterprétation nom > particule d'extension. Comme on l'a déjà annoncé plus haut, l'étude de nos données nous conduira à prendre des distances par rapport au scénario diachronique de «pragmaticalisation» postulé par Mihatsch dans son article de 2006.

28. Pour les détails sur nos données et sur la méthodologie adoptée, voir la section I.2.

29. Il ne faut pas chercher d'isomorphie avec les types dégagés supra (§ 2.3) à propos de ou comme ça, dont le statut morphosyntaxique et sémantique est, on l'a souligné au début, fort différent. 


\subsection{Statut sémantique de machin}

Le substantif machin fait partie, avec chose, truc, bidule, fourbi et quelques autres, d'une série de termes dits «vagues» qui, en raison de leur sémantisme sous-spécifié, compatible avec un très grand nombre de référents, ont aussi été qualifiés de «postiches», «vicaires», «relais» (Kleiber, 1987: II2) ou "passe-partout» (Mihatsch, 2006: $153^{30}$; Halmøy, 2006); leur fonctionnement est parfois comparé à celui des indéfinis (Muller, 20I0; Schnedecker, 20II). Dans un texte de 1927, l'écrivain Duhamel les appelle joliment des «mots omnibus»:

[22] Il n'emploie presque jamais les substantifs convenables; il les remplace par deux ou trois mots omnibus: «vous voyez ce truc? C'est absolument comme un macbin...» et il s'étonne, si l'on ne comprend pas.

(Frantext: G. Duhamel, Le journal de Salavin [1927], Paris, Mercure de France, I947, p. 74)

L'auteur se fait ici l'écho des préjugés normatifs qui pèsent (sans beaucoup de nuances) sur ces noms postiches, dont les vertus en discours sont pourtant évidentes, comme il apparaîtra dans la suite.

Notre but n'est pas ici de chercher à cerner les limites du paradigme constitué par les noms vagues, ni de caractériser, dans la foulée de l'étude pionnière d'Halmøy (2006), leurs fonctionnements respectifs. Pour mener à bien un tel travail, il conviendrait d'analyser, dans de vastes corpus relevant de genres diversifiés, les distributions respectives de chose, truc, machin, bidule et autres, qui commutent dans certains contextes mais non dans d'autres (voir Delafontaine, 2016).

Machin, qui nous retiendra seul ici, est rapproché par les historiens de machine, dont il semble avoir été tiré par dérivation inverse ${ }^{31}$. Marqué (au même titre que truc et bidule) par un trait de familiarité, il n'apparaît dans les textes que de manière tardive: le premier exemple fourni par Frantext ${ }^{32}$ figure dans une correspondance de Victor Hugo publiée en $\mathrm{I}_{42^{3}}{ }^{33}$. Bien que le terme connaisse ensuite un certain essor, il demeure infiniment plus rare à l'écrit que chose qui, présent dès les premiers témoignages du français, est stylistiquement neutre et intégré à un grand nombre de

30. Voir l'étude de Fronek (I982) à propos de l'anglais thing.

31. Ce lien est clairement ressenti par les locuteurs francophones, ce qui transparait dans des figures étymologiques comme celle-ci:

[vii] [...] des vaudevillistes, des dramaturges, le fameux machin auteur de plusieurs grandes machines. (Frantext: A. Daudet, Fromont jeune et Risler aîné [1874], Paris, Gallimard, 1986, p. 952)

[viii] Et la machine le trompe avec un machin un machin à mourir de rire.

(Frantext: J. Prévert, La pluie et le beau temps [1955], Paris, Gallimard, 1998, p. 102)

32. Base consultée pour la dernière fois en décembre 2016 .

33. Le voici :

[ix] Comme ils avaient invariablement oublié le nom des personnages dont ils parlaient, l'un disait M. Chose, et l'autre M. Machin. Ils se comprenaient. (Frantext: V. Hugo, Le Rhin: lettres à un ami [1842], Paris, P. Ollendorff, 1906, p. 236) 
locutions figées ou semi-figées ${ }^{34}$. À noter que parmi les noms vagues, seul machin semble développer de façon claire, de nos jours, un emploi de particule d'extension. Cette acception, sans doute à cause de son caractère familier et récent, n'est pas répertoriée dans le $T L F i$, selon qui machin est un substantif utilisé:

Pour désigner qqc. ou qqn dont on ne connait pas ou dont on ne se rappelle pas le nom; pour remplacer le nom de qqc. qu'on se refuse, ou qu'on néglige de nommer clairement.

(TLFi, s. v. «Machin»)

Dans sa fonction de «joker» de nom propre, machin alterne souvent avec les autres termes vagues, surtout chose et truc, pour désigner des référents distincts (exemple [23]). Il peut aussi former avec eux un nom composé désignant un référent unique (exemple [24]) (voir Schnedecker, 2oII):

[23] MACHIN, TRUC, CHOSE, tous morts, tous tués, crevés, écrabouillés, anéantis, disloqués, oubliés, pulvérisés, réduits à zéro.

(TLFi: B. Cendrars, La main coupée, Paris, Denoël, 1946, p. 314)

[24] - Comprends pas: moins il y a d'émissions littéraires à la télévision, plus on invite de gens de la télé aux cocktails littéraires. Lui, là, par exemple, Machin-Chose, le sinistre rigolo de la météo, on l'a invité pour empêcher qu'il pleuve ce soir, peut-être [...].

(Frantext: J.-L. Benoziglio, La voix des mauvais jours et des chagrins rentrés, Paris, Seuil, 2004, p. 123-124 ${ }^{35}$ )

Macbin se prête en outre - toujours selon le $T L F i$ - à «désigner une chose, une œuvre, une institution dont on parle avec ironie (réelle ou feinte). Machin diplomatique, philosophique; machin en vers» ${ }^{36}$. Les exemples livrés par Frantext, qui mériteraient d'être analysés en détail, s'appliquent à des objets concrets, à des êtres humains, des paroles, des souvenirs, voire des actions diverses (en particulier suite à un verbe de type faire)...

34. Voir grand-chose, quelque chose, autre chose (Halmøy, 2006). La recherche de la forme fléchie machin dans Frantext livre 840 résultats bruts. Certains d'entre eux relèvent d'un patronyme Machin qui préexiste à notre lexème (selon Dauzat [1977: 248], il s'agit d'une altération de Martin d'origine basque), et qui en a sans doute été rapproché. Cela a pu favoriser l'essor des emplois comme celui de Victor Hugo cité dans la note qui précède. Quant aux occurrences de chose, elles sont si nombreuses dans Frantext qu'elles excèdent les limites de requête de la base, fixées à 50000 occurrences.

35. Voir, dans ce même emploi, machin-chouette. On peut relever que machin(-chose) occupe parfois la place non d'un signe, mais d'une simple séquence syllabique:

$[\mathrm{x}]-[\ldots]$ Tu m'en parlais de mon grand-père d'Istan-machin-chose?

- Is-tan-bul. Non, pas vraiment...

(Frantext: J.-L. Benoziglio, Cabinet portrait, Paris, Seuil, 1980, p. 217)

Par ailleurs, une des informatrices intégrées plus récemment dans OFROM fournit deux exemples de la séquence machin bidule en fin de liste. En voici un:

[xi] ah c'est pour des recherches sur euh + plein de trucs quoi linguistique euh machin bidule (unine16-018)

36. Le TLFi rappelle que le général de Gaulle désignait par le Machin l'Assemblée générale des Nations Unies. 
En discours, les SN contenant machin permettent d'introduire un nouveau référent de manière nonchalante (Berrendonner, 1990), c'est-à-dire en s'épargnant la recherche d'un éventuel «mot propre» (voir l'exemple [25]); ils permettent aussi de rappeler de manière économique, en s'appuyant sur les capacités inférentielles de l'interlocuteur, un référent préalablement validé dans la mémoire discursive (exemple [26]):

[25] Qu'y a-t-il donc là-dedans [dans le vin cuit]? [...] Toutes sortes de choses! [...] d'abord des machins qui viennent des Indes, la cannelle, des herbes qui vous changent, par enchantement.

(TLFi: H. de Balzac, Les paysans [1844-1850], Paris, 1955, p. 207)

[26] - Vous n'avez pas essayé le Braille?

- Vous voulez dire, ces trucs avec les points?

- Oui.

- Non. À l'hôpital, on voulait m’apprendre ça. Mais c'est trop compliqué, ce machin-là.

(Frantext: J.-M. G. Le Clézio, Le déluge, Paris, Gallimard, 1966, p. II5)

Il arrive que le contexte discursif fournisse, directement ou indirectement, une désignation plus précise du référent. Mais la tâche d'identification peut aussi être laissée entièrement à la charge de l'interprète. Ainsi dans l'exemple [27], qui illustre le rendement euphémique bien connu des noms vagues:

[27] - ... Le petit machin de bébé est très enflé, Madame..., c'est très rouge. - Je sais, lui répondit sèchement Marie; laissez-nous.

(Frantext: A. Memmi, Agar [1955], Paris, Gallimard, 1984, p. 144)

Le corpus oral OFROM contient à la fois des emplois conformes aux types illustrés ci-dessus, et des emplois d'une autre nature comme les suivants:

[28] pas contents de me voir mais je veux dire ils disent ah génial un jeune machin on pourra sortir avec lui

(unineı2-daa)

[29] ouais je devais [ $\mathrm{t}]$ être ado et je me rappelle elle avait donné un grimoire à ma grandmère un joli machin + mais maintenant je connais bien mais avec de l'écriture du quinzième siècle dedans + machin qui est de recettes de toubib et cetera qui est- elle le lisait encore la bonne dame + ma grand-mère «moi je comprends rien» elle ar«mais t'a-» et pis elle lui disait attends comment elle s'appelait + pas Noémie mais + «t'arrives à lire» «ah ouais ouais» pis elle lisait + machin les recettes pis tout dedans (unifrII-dba)

Dans l'exemple [28], on pourrait croire, à lire la transcription, à un emploi substantival, où machin serait nom-tête d'un $\mathrm{SN}$ : un jeune machin. Mais l'écoute de l'extrait exclut, sans doute possible, une telle interprétation : c'est jeune qui est ici le nom-tête, et macbin, prononcé de manière détachée, avec un contour intonatif 
copiant ceux qui ont été réalisés successivement sur ah génial et un jeune (voir Johnsen et Avanzi, en prép.), assume, comme c'était le cas dans les exemples [19]-[2I], une fonction de prolongateur.

Quant à [29], machin y apparaît à trois reprises. Les deux premières occurrences (joli machin, machin qui...) sont nominales et correspondent aux définitions du TLFi. Dans la troisième en revanche, il semble fonctionner comme une sorte de marqueur d'indétermination (pour dire les choses un peu vite). Les trois emplois partagent un point commun: ils sont associés à des phénomènes de liste au sens large (liste appositive comme dans un grimoire... un joli machin... macbin qui...; énumération - ou du moins ébauche d'énumération - dans machin les recettes pis tout dedans).

\subsection{Typologie des emplois de machin relevés dans la base OFROM}

Sur une base distributionnelle, il est possible de distinguer dans nos données trois types d'emplois nominaux de machin, et trois types d'emplois en tant que particule d'extension ou d'indétermination.

\subsubsection{Type 1: joker de nom propre, nom postiche}

Le type I recouvre les emplois substantivaux traditionnels et bien connus de notre lexème (voir la section 3.I). Machin y sert soit de nom postiche occupant la position d'un nom propre, soit de nom commun «omnibus». Dans ces deux cas, il est utilisé tantôt pour pallier une lacune lexicale, due à l'ignorance ou à un trou de mémoire du locuteur (exemples [30]-[3I]), tantôt pour désigner, de manière nonchalante et avec un effet de connivence, un référent que le contexte discursif permet d'identifier aisément (exemple [32], voir déjà l'exemple [29] ci-dessus):

[30] ah j’ai été voir le spectacle de machin chouette (uniners-029)

[3I] oh je sais que c'est la commune mixte de machin truc (uniners-065)

[32] pis les bistrots sont différents + tu vas dans en en en en au Cardoche en haut tu vas le le boire un pot + ciao tu te mets à une table $\%+$ on $s^{-}+$on on y a jamais un gars tout seul qui prend son pot + en bas tu vas dans un machin tu prends ton truc tu $\mathrm{t}-$ tu y a pas d- $\mathrm{y}$ a moins de + de de de liant entre les gens (unineı5-or8; dans cet extrait, «\%» note un segment inaudible)

Dans l'exemple [32], le locuteur est engagé dans une comparaison entre les modes de sociabilité qui caractérisent le «haut» et le «bas» du canton de Neuchâtel ${ }^{37}$. La séquence «tu vas dans un machin tu prends ton truc», en fonction de l'état

37. En gros, et selon le stéréotype ambiant, la chaleur de l'accueil en milieu montagnard, par opposition à la froideur des habitants du bord du lac. 
des connaissances partagées, peut être interprétée en gros comme «tu vas dans un bistrot, tu prends ton pot...». Une paraphrase qui se borne à introduire une redondance au plan lexico-sémantique a néanmoins quelque chose d'insatisfaisant. Elle ne rend pas justice au décrochement générique instauré par l'usage des deux noms vagues qui, dans le contexte concerné, peuvent s'appliquer à différents types d'établissements publics (bars, mais aussi restaurants, dancings...) et à différents types de consommations (solides autant que liquides).

\subsubsection{Type 2: nom postiche + spécification lexicale}

Le substantif postiche macbin apparaît en outre, comme l'ont observé BlancheBenveniste et Jeanjean (1988: I7I), dans une routine de spécification lexicale où le nom vague apparait en première position. Cette routine est à l'œuvre dans les exemples [33] et [34] où, pour pointer ou introduire un référent, un lexème vague fait place, dans une structure de liste, à un désignateur plus précis:

[33] et pis + où y a les raisinets ben ma foi y a y a ces machins-là + ces frênes qui repoussent

(uniner5-023)

[34] Li: bon le problème c'est les micros là qui sont pas euh protégés + je sais pas si tu + si y a un machin à mettre par-dessus tu sais anti euh vent +

$\mathrm{L}_{2}: \mathrm{mh} \mathrm{mh}+$ des bonnettes

(uniners-06r)

Dans l'exemple [33], l'apport du désignateur le plus riche en sèmes figure dans le même tour de parole. Mais à d'autres occasions (exemple [34]), c'est l'interlocuteur qui est appelé à la rescousse pour faire aboutir la recherche lexicale (voir le rôle ici de $t u$ sais). L2 est alors amené à formuler le nom technique dont l'hyperonyme corrigé macbin anti-vent fournit une première approximation. Après avoir acquiescé $(m b m b), L_{2}$ fournit le terme attendu en prenant appui sur le matériau syntaxique de l'intervention précédente (noter le passage au pluriel: des bonnettes).

\subsubsection{Type 3: nom postiche en fin de liste}

Chez les locuteurs du corpus OFROM, la routine de recherche lexicale décrite à la section précédente est cependant rare. Et bien plus souvent, le nom postiche intervient à la fin d'une liste de SN, d'adjectifs, d'infinitifs, dont il participe, quoique de manière vague, à l'élargissement:

[35] moi j’y ai fait toute mon enfance avec eux dans le jardin + à récolter des légumes des trucs des machins

(uninerz-hma)

[36] c'est des espèces de + de villes semi-touristiques semi-bidonvilles semi-machin (uninerz-hma) 
[37] quand t'as des gamines qui viennent te dire qu'elles se sont fait violer à la maison ou euh + taper ou machin

(unineo8-hda)

[38] y a des mots différents + je crois dans 1- dans le Jura + ils appellent un fer à repasser une beuglisse + des machins comme ça

(uniners-029)

[39] tu sais c'est genre quoi euh je je vais filmer quatre jours euh + huit heures de d- de de théorie de travail de groupe de machin et tout pis je dois faire un film de trois minutes (unifriı-maa)

Le ou les élément(s) lexicalement spécifié(s) figure(nt) alors en tête de liste, et le SN contenant machin vient clore l'énumération. La manœuvre de prolongation consiste à aller du spécifique au vague, dans un mouvement de généralisation ou d'indistinction (voir l'exemple [32] ci-dessus). Dans cette fonction, le SN avec machin est parfois doublé par un SN contenant un autre nom vague (exemple [35]); et comme dans les exemples [20] et [2I] supra, il peut être accompagné d'un extenseur tel que et tout (exemple [39]). Par ailleurs, on peut noter qu'il s'adjoint sans difficulté à des épithètes adjectivales (exemple [36]) ou à des infinitifs (exemple [37]).

Les exemples du type 3 présentent certaines analogies avec les séquences ou comme ça des sections 2.2 et 2.3. Ainsi, dans l'exemple [35], la formule des trucs des machins enchaîne sur un SN désignant un produit de récolte prototypique (des légumes), et l'on aurait probablement aussi pu rencontrer, dans la bouche de notre locuteur, des légumes ou comme ça; autres points de rencontre: dans l'exemple [37], le lexème vague est précédé de ou et dans l'exemple [38] machins est déterminé par comme ça.

Cependant, quand il est assuré par recours à un nom vague, l'élargissement d'un paradigme a plus souvent lieu sur le mode de la coordination implicite que sur celui de l'alternative. Et - différence majeure avec ou comme ça, et tout, et tout ça- le recours au nom vague permet seul de maintenir ne varietur le moule syntaxique instauré au préalable: voir semi- $X$, semi- $Y$, semi- $Z$ dans l'exemple [36]. Une formule du type ou comme ça ne pourrait guère ici, sans effet de rupture, occuper la position de Z, alors que machin s'y trouve au contraire parfaitement intégré.

Grâce à ce statut de joker, machin permet ainsi de prolonger une liste sans rompre le parallélisme syntaxique de ses constituants. Dans l'exemple [36], semi-machin s'adjoint à une énumération semi-touristiques semi-bidonvilles dont on pourrait penser qu'elle a épuisé le paradigme (du moins si l'on comprend le préfixe semi- comme signifiant «à moitié»). En l'absence de toute marque d'hésitation, il apparaît peu probable que semi-machin serve ici à suppléer un «vide lexical» que l'interlocuteur serait invité à combler ${ }^{38}$. Si, dans son élan énumératif, le locuteur rajoute semi-machin,

38. Mihatsch (2006) voit là la fonction par excellence du mot passe-partout machin, mais il nous semble que cette vue est réductrice et souvent démentie par les faits. 
on peut penser que c'est pour renforcer à peu de frais, en ajoutant un troisième élément, le potentiel argumentatif d'une liste d'épithètes censée accréditer le caractère hybride et peu plaisant des localités dont il est question. L'intérêt stratégique que le locuteur peut avoir à prolonger une liste transparaît aussi dans l'exemple [39], reproduit ici sous forme de grille:

[39'] je vais filmer quatre jours euh

huit heures de

d

de

de théorie

de travail de groupe

de machin

et tout

Dans cet exemple, de machin et et tout permettent de donner plus de corps à une énumération initiée avec une certaine peine (voir les piétinements sur la préposition $d e$ ) et qui, en l'absence de prolongateurs, risquerait d'apparaittre quelque peu étriquée. Or, le locuteur tient visiblement dans ce passage à mettre en valeur le travail de longue haleine que nécessite la production de trois brèves minutes de film. Son ambition emphatique est sensible, à l'écoute, dans une prosodie créatrice d'attentes, qui allonge à l'extrême les voyelles finales des mots théorie, groupe et machin ${ }^{39}$.

\subsubsection{Type 4: emploi comme particule d'extension après une énumération}

Avec le type 4, on s'éloigne davantage encore des emplois répertoriés par les dictionnaires. Ici, macbin apparaît sans déterminant, et sans qu'une préposition soit répétée devant lui quand le cas pourrait se présenter (comparer travail de groupe de machin, type 3 , à travail de groupe machin, également possible, et qui relèverait du type 4). Comme on l'a mentionné plus haut, les transcripteurs traitent alors le terme comme un mot invariable (voir l'exemple [44]), et le qualificatif de particule d'extension semble adéquat pour caractériser cet emploi:

[40] on n'a que des shorts on n'a pas besoin d'avoir des doudounes des habits de ski des habits pour comme protection contre la pluie des bottes machin on a que des tongs et pis des shorts

(uniner5-037)

[4I] il a reçu la ferme il a reçu machin (uniners-or8)

39. Les phénomènes de parallélisme intonatif sont très sensibles dans la version audio de nos exemples et fort intéressants à observer: on ne peut ici que le signaler rapidement, en invitant le lecteur à aller écouter les sons disponibles sur le site de la base OFROM. 
[42] avec les gars qu'ils ont choisis et tout machin (unifriı-maa)

[43] j'ai un diplôme un diplôme fédéral de + de moniteur de musicien machin enfin + tous les trucs

(unineII-smb)

[44] tu sais euh ils travaillent avec des post-it machin ils font des grands dessins sur des murs

(unifriı-maa)

[45] ouais après ben le le y a le parachute qui s'ouvre machin on atterrit (uniner2-avb)

Dans les items de cette série, machin vient parfois clore une liste énumérative en laissant entendre qu'elle pourrait être prolongée, mais sans gain notable du point de vue de la pertinence informationnelle (des doudounes des babits de ski des babits pour comme protection contre la pluie des bottes machin); voir la section 2.3 supra. Il peut aussi amorcer la clôture d'une liste, comme dans l'exemple [43] où il est suivi de enfin tous les trucs. Mais il arrive aussi qu'il figure au sein d'une liste de prédicats, où il permet d'évoquer de manière elliptique une phase jugée subsidiaire d'un script ou d'un scénario dont le récit explicite peut être repris ensuite si nécessaire (exemples [44] et [45]). Dans les exemples ci-dessus, machin est inséré aussi bien là où on attendrait un SN complément du verbe (il a reçu la ferme il a reçu machin, qui laisse inférer «il a reçu plein de choses»), qu'en lieu et place d'une clause indépendante (exemples [44] et [45]). À noter, ici encore, que les phénomènes de scansion et les parallélismes intonatifs sont très présents dans les versions sonores de ces exemples.

\subsubsection{Type 5: emploi comme particule d'extension ou d'approximation après un discours rapporté}

Une position privilégiée occupée par la particule machin chez certains locuteurs, également relevée par Halmøy (2006) et par Secova (2014) dans les corpus faisant intervenir de jeunes Parisiens qu'elle a étudiés, c'est la finale de discours rapportés. Nous avons observé cela pour des discours directs, indirects et directs libres (ou mentions-échos). À l'instar de ce que nous venons de voir à propos du type 4 , machin ne figure pas forcément à la toute fin du discours rapporté: il peut être suivi d'une relance, comme dans l'exemple [48], où il est en outre redoublé.

[46] je l'avais appelé le jour avant pour lui pour voilà pour lui dire ben j’espère que ça va bien se passer bonne chance machin

(unineo8-vwa)

[47] ils nous font ah ben on va dans l'avion machin donc euh on nous on s'y attendait trop pas

(uniner2-avb) 
[48] elle dit ah non non alors j'aimerais savoir tout de suite machin machin on peut pas + je peux pas euh je peux pas me permettre de perdre du temps comme ça (uniners-064)

[49] pis après il voulait savoir qui c'était ce mec euh machin euh (unineo8-vwa)

Il s'agit là d'un emploi proche de celui qui a été décrit dans la rubrique précédente, et dont nous avons trouvé des adaptations écrites sur la toile:

[5०] «C'est un mec trop normal», confie Léonard Trierweiler. «Parfois à l'Élysée ça lui arrivait d'éteindre lui-même les lumières, genre "ça coûte trop cher machin..." [...]». (http://www.bfmtv.com/)

L'usage de la particule, attribué selon toute apparence au discours cité (alors que ou comme ça, en même position, marque un retour au discours citant), permet d'épargner des développements qui pourraient s'avérer oiseux et peu pertinents. Il s'agit d'une façon familière d'indiquer que l'auteur du discours cité a parlé à peu près en ces termes, mais que l'on peut avantageusement se passer de rapporter ses propos plus en détail.

\subsubsection{Type 6: en tête de liste, avec fonction d'approximateur}

Enfin, dernier type plus rare, relevé à deux reprises seulement dans OFROM : c'est le cas où machin apparaît non pas en fin de liste, mais au début, donc plutôt en tant qu'amorce. C'est le cas de la première occurrence du terme dans [5I] ou de l'occurrence présente dans l'exemple [28] vu plus haut. On est ramené ici, en quelque sorte, à une routine de spécification progressive.

[5I] tu sais machin tu sais genre les gars stressés qui discutent à fond entre eux qui font des grands signes et tout + avec une petite musique machin + tu sais genre un peu l'aperçu genre limite ralenti

(unifrı-maa)

L'exemple [5I] est intéressant, avec les trois tu sais faisant appel aux compétences inférentielles de l'interlocuteur, et avec une accumulation de marques d'indistinction et d'approximation: machin, genre, un peu, limite... (voir la section 2.2.4).

\subsubsection{Emplois ambigus}

83

À noter aussi que dans certains cas, l'analyste peut hésiter: machin est-il employé en tant que nom ou en tant que particule d'extension (exemples [52]-[54]) ? Et dans l'exemple [55], faut-il voir un postiche occupant la place d'un constituant régi, ou s'agit-il d'un prolongateur indiquant que le discours direct ne rapporte qu'en substance et de manière approximative?

\section{[52] des évaluations A B C machin (unifrII-086)}


[53] on avait fait une bouffe euh chez lui euh chez sa mère + avec ses potes et pis euh voilà quoi + enfin euh grillades euh machin(s)

(unineo8-vwa)

[54] ça m'attirait absolument pas + monter s'entraîner machin dans [interruption] (uniners-org)

[55] parce qu'en fait j'ai croisé une copine qui voulait du enfin qui cherchait du travail pis je lui ai dit ben je vais appeler + euh voir s'il a besoin machin

(unineo8-vwa)

La prise en compte de ces contextes analysables de deux, voire de plusieurs manières, est importante à la fois pour rendre compte de la connexité du système, c'est-à-dire des facteurs qui relient les emplois nominaux aux emplois de type particule d'extension, et pour comprendre comment ceux-ci ont pu se développer à partir de réinterprétations locales (voir 3.4).

\subsection{Synthèse des emplois}

Le tableau 2 ci-dessous est un tableau de synthèse résumant les observations qui ont été faites en 3.2. Y figure également le nombre d'occurrences relevées pour chaque type d'emploi.

Les emplois nominaux univoques sont au nombre de 56 , et ne représentent donc que $57,7 \%$ des attestations du lemme machin dans notre corpus. Quant aux emplois univoques en tant que particule, ils se montent à 30 , ce qui équivaut à près d'un tiers des emplois $(30,9 \%)$. Entre deux figurent les items que nous avons estimés ambigus (II exemples, soit II, $3 \%$ ). Ils concernent principalement des cas où machin peut être interprété soit comme nominal, soit comme particule d'extension (voir 3.2.7).

En résumé, quand il figure dans des listes, le nom machin semble apte à servir deux types de routine inégalement représentés dans nos données: (i) l'une où le locuteur, dans une démarche de recherche lexicale, cherche à spécifier progressivement un référent ou un ensemble de référents (ces machins-là ces frênes); (ii) l'autre où le locuteur, suite à la mention d'un ou de plusieurs référents spécifiés, rajoute un SN contenant un lexème-postiche qui lui permet de suggérer, dans un style informel, un élargissement du paradigme, de manière à la fois vague, économique et non directive du point de vue interactionnel (d'où un effet de connivence et un rôle mitigateur: des légumes des trucs des machins). Nous avons toutefois déjà relevé plus haut la faible représentation de la «spécification lexicale progressive» (2 occurrences seulement) par rapport à l'«ouverture paradigmatique» (19 occurrences).

Plus globalement, on peut noter que 62 occurrences de macbin, tous emplois confondus (soit $63,9 \%$ de l'ensemble), mettent en jeu des phénomènes de listes, énumératives ou appositives, à deux ou à plusieurs termes (voir à ce propos la section 4). À la différence des séquences et tout, et tout ça, et de ou comme ça examiné 


\begin{tabular}{|c|c|c|c|}
\hline $\begin{array}{l}\text { Type } \\
\text { d'emploi }\end{array}$ & Exemple & Aspect fonctionnel remarquable & $\begin{array}{l}\text { Nombre } \\
\text { d'occurrences }\end{array}$ \\
\hline Type I & $\begin{array}{l}\text { tu vas dans un machin tu } \\
\text { prends ton truc }\end{array}$ & $\begin{array}{l}\text { Nonchalance dans la désignation, } \\
\text { économie lexicale. }\end{array}$ & 35 \\
\hline Type 2 & $\begin{array}{l}\text { y a ces machins-là }+ \text { ces } \\
\text { frênes qui repoussent }\end{array}$ & $\begin{array}{l}\text { Recherche lexicale, routine de } \\
\text { spécification progressive. }\end{array}$ & 2 \\
\hline Type 3 & $\begin{array}{l}\text { récolter des légumes des } \\
\text { trucs des machins }\end{array}$ & \multirow{3}{*}{$\begin{array}{l}\text { Ouverture paradigmatique, avec } \\
\text { ou sans effet d'emphase. }\end{array}$} & 19 \\
\hline Ambigus & $\begin{array}{l}\text { enfin euh grillades euh } \\
\text { machin(s) }\end{array}$ & & II \\
\hline Type 4 & $\begin{array}{l}\text { avec les gars qu'ils ont } \\
\text { choisis et tout machin; } \\
\text { y a le parachute qui s'ouvre } \\
\text { machin on atterrit }\end{array}$ & & 18 \\
\hline Type 5 & $\begin{array}{l}\text { ils nous font ah ben on va } \\
\text { dans l'avion machin }\end{array}$ & $\begin{array}{l}\text { Économie discursive, approxima- } \\
\text { tion (post-DR). }\end{array}$ & I0 \\
\hline Type 6 & $\begin{array}{l}\text { machin les recettes pis } \\
\text { tout dedans }\end{array}$ & Approximation, amorce de liste. & 2 \\
\hline Total & & & 97 \\
\hline
\end{tabular}

Tableau 2 - Synthèse des emplois de machin(s) relevés dans le corpus OFROM

en 2, la particule d'extension machin se révèle relativement mobile; elle se trouve même à l'occasion en tête de liste, dans une position où figurent également genre ou style. Elle prend alors un sens d'approximation ou d'indétermination.

\subsection{Les conditions syntaxiques d'une réinterprétation}

Dans le corpus examiné, le terme machin est, on l'a vu, polyfonctionnel. Cependant, nos données ne permettent pas de confirmer le canevas évolutif proposé par Mihatsch (2006). Selon la chercheuse, le nom passe-partout machin posséderait «une force déictique particulière demandant à l'auditeur de [le] remplacer [...] par un nom spécifique à l'aide du savoir contextuel» (Mihatsch, 2006: 153). Et le mot aurait été soumis à un scénario de "pragmaticalisation» qui, à partir d'un sens descriptif et via un processus de "subjectification", l'aurait conduit à devenir un marqueur pragmatique d'hésitation (Mihatsch, 2006: 159 et 167).

Cette hypothèse n'est pas confirmée par nos données. Tout d'abord, en discours, l'emploi du nom vague n'intervient pas seulement pour couvrir l'indisponibilité d'un «nom adéquat» (Mihatsch, 2006: 155), mais aussi bien souvent, on l'a vu plus haut, pour permettre une ouverture paradigmatique, une généralisation. En ce cas, il n'est pas particulièrement attendu de l'auditeur qu'il «remplace» le nom vague par un nom spécifique. 
D'autre part, si l'on en croit nos données, machin fournit au français contemporain non pas un marqueur d'hésitation, mais bel et bien une particule d'extension (non repéré par Mihatsch, ce rôle est décrit chez Halmøy [2006], Johnsen [20II] et Secova $[2014]^{40}$ ).

Certes, comme le montre l'exemple fourni dans le tableau 2 pour illustrer nos exemples ambigus (enfin eub grillades eub machin(s)), il n'est pas rare que machin côtoie des signaux d'hésitation. Mais cela n'implique pas qu'il soit en lui-même un marqueur d'hésitation; bien souvent d'ailleurs, il est prononcé avec aplomb et sans le moindre signe de tâtonnement, ainsi par exemple dans nos extraits [36] ou [48].

Pour nous, la polysémie de machin ne gagne pas être étudiée «hors-sol», en fonction d'un scénario d'évolution sémantique préconçu. En réalité, le double statut de machin (nominal et comme particule d'extension) prend naissance dans des conditions morphosyntaxiques bien précises. Envisagées sans sélection a priori, nos données nous enseignent que c'est l'aptitude de machin à figurer en fin de liste, et non la gestion de difficultés d'accès lexical, qui sert de creuset à une transcatégorisation; l'essor de macbin comme particule d'extension s'explique d'abord et avant tout par la propension des noms vagues à être utilisés comme indicateurs d'ouverture d'un paradigme quelconque (des $\mathrm{X}$, des $\mathrm{Y}$, des trucs des machins). À la fin d'une liste constituée d'infinitifs (violer, taper, machin), de noms sans déterminant (grillades machin(s)) (voir 3.2.7), ou dans le cadre d'une période-liste ( $y$ a le parachute qui s'ouvre macbin on atterrit), le nom vague apparait tout nu et se prête à être réinterprété comme une particule invariable. Utilisée à la fin d'un discours rapporté, ladite particule peut à son tour se charger d'un sens d'approximation, et acquérir de ce fait une mobilité syntaxique nouvelle (voir 3.2.6). Il n'y a lieu de parler ici ni de blanchiment sémantique, ni de décatégorisation, encore moins de «subjectification»: le changement constaté s'explique par une simple réanalyse syntaxique, qui modifie le rôle de macbin dans la liste qui l'accueille, tout en entraînant une réinterprétation catégorielle.

\section{Conclusions}

Les marqueurs ou comme ça et machin présentent (i) des points communs et (ii) des différences que l'on peut synthétiser comme suit.

(i) Outre le fait qu'ils assument tous deux, entre autres, des fonctions d'extenseurs, et qu'ils ont des rendements pragmatiques communs (approximation ${ }^{41}$, discours rapporté «en substance»), ils partagent la fonction interpersonnelle d'ouverture paradigmatique (à la source d'un effet de «discours moins catégorique») et contribuent

40. Cette dernière parle toutefois, à propos de machin, de décatégorisation (Secova, 20I4: 287) là où il y a clairement pour nous recatégorisation.

41. Une approximation qui n'est cependant pas du même ordre. Ainsi, machin, qui est plus commun comme approximateur que ou comme ça, n'apparaît pas après un chiffre (voir supra, en 2.2.I). 
à établir une connivence entre les interlocuteurs (Johnsen, 20II; Secova, 20I4; Gadet, 20I7). L'emploi comme extenseur remplit ainsi un rôle préventif tout en permettant une économie - c'est une opération que l'on s'épargne.

(ii) Du côté des différences, nous avons déjà souligné le caractère compositionnel de ou comme ça et mono-morphématique de machin. L'indétermination réside, pour machin, dans sa valeur lexicale (traditionnellement, postiche de nom propre ou de nom en général; mais aussi postiche de clause et d'autres catégories), alors que pour ou comme ça elle est liée à la fois à un élément zéro souplement récupérable, au ou qui permet d'évoquer des référents alternatifs, ainsi qu'au ça qui pointe un référent non individué. Surtout, le caractère indivisible de machin le rend nettement plus mobile et favorise sa recatégorisation comme particule d'extension et d'approximation.

Dans un autre registre, notre étude questionne (i) la définition du phénomène d'énumération et (ii) la problématique du vague dans les langues naturelles.

(i) Il est courant qu'un lien soit établi entre énumération et nombre minimum d'items. En voici trois illustrations:

Une question importante pour la définition de l'énumération concerne le nombre minimum d'items requis. Sur cette question, les avis divergent. Certains travaux considèrent qu'il y a énumération à partir de deux items (Turco et Coltier, 1988). Pour d'autres, il est nécessaire d'avoir au moins trois items. Schnedecker (1998) par exemple inscrit les corrélats anaphoriques comme l'un/l'autre, d'une part/d'autre part, le premier/le second, soit... soit, tantôt... tantôt en dehors du champ des structures énumératives.

(Bras et al., 2008: 1955)

Adam (1990) rapporte cette définition de l'énumération tirée d'un ouvrage de Damamme-Gilbert intitulé La série énumérative (1989):

Nous appellerons «série énumérative» toute expression linguistique formée d'un nombre minimum de trois termes (mots, syntagmes, unités d'énoncé) qui appartiennent à des catégories morphologiques ou grammaticales identiques ou équivalentes, qui occupent une fonction identique dans la syntaxe de l'énoncé et qui, placées côte à côte, sont coordonnées ou reliées par un signe de ponctuation. (Adam, 1990: I5I)

Enfin, Bonvino et al. (2009: I) définissent la liste comme: «a combination of two or more units of the same type realizes one and the same constructional slot».

Les données orales qui présentent un extenseur comme ou comme ça ou machin rendent un tel dénombrement relativement vain. En effet, les faits linguistiques soumis à l'analyse nous conduisent plutôt à considérer - suivant en cela Johnsen (20II) et Secova (20I4: 28I) - qu'une énumération peut ne contenir qu'un seul élément lexical, accompagné d'au moins une particule d'extension : et tout, tout ça, ou comme ça, machin. 
(ii) Si l'énumération n'est souvent qu'esquissée, les protagonistes de l'échange verbal s'accommodent parfaitement de cette situation. Qui plus est, ces particules d'extension rendent de grands services aux locuteurs, en leur permettant de ne pas entrer dans les détails quand cela n'est pas essentiel, de ne pas s'engager trop catégoriquement dans leur discours, de laisser à l'allocutaire une latitude d'interprétation. Nos résultats sont ainsi convergents avec ceux de Jucker et al. (2003), pour qui les marqueurs d'indétermination ne sont pas moins pertinents (en termes de coûts et d'effets) que des formulations plus précises.

En observant les corpus oraux, on se rend compte que pour les locuteurs, il n'est pas toujours aisé de formuler une énumération exhaustive - ni dans d'autres cas, pour des raisons argumentatives, de mettre un terme à une énumération même plus ou moins saturée; les moyens linguistiques à disposition pour prolonger les listes remédient opportunément à ces difficultés. Les stratégies discursives adoptées, qui mettent à profit les termes et les structures à sens vague, ne sont pas problématiques, dans la mesure où locuteurs et interlocuteurs s'accommodent parfaitement d'une part d'indétermination dans la désignation.

\section{Références}

Adam, J.-M. 1990. Éléments de linguistique textuelle. Liège: P. Mardaga.

Authier-Revuz, J. 1995. Ces mots qui ne vont pas de soi. Boucles réflexives et non-cö̈ncidences $d u$ dire. Paris: Larousse.

BÉGUELIN, M.-J. 20I6. Quand la référence se fait évanescente : ne pas en démordre, gros comme ça et autres locutions à pointeurs démotivés. In L. SARDA, D. Vigier et B. COMBETTES (éd.), Connexion et indexation. Ces liens qui tissent le texte. Lyon: ENS éditions: 99-I2I.

BerRendonner, A. 1990. Attracteurs. Cabiers de linguistique française II : I49-158.

BILGER, M. 1982. Contribution à l'analyse en grille. Recherches sur le français parlé 4: 195-215.

BILGER, M. 1989. Les réalisations de et tout (ça) à l'oral. Recherches sur le français parlé 9 : 97-IO9.

Blanche-Benveniste, C. 1987. Syntaxe, choix de lexique et lieu de bafouillage. DRLAV 36-37: $123-157$.

Blanche-Benveniste, C. 20ir. Les beautés de l'énumération. In G. Corminboeuf et M.-J. BÉGUELIN (éd.), Du système linguistique aux actions langagières. Mélanges en l'honneur d'Alain Berrendonner. Bruxelles: De Boeck: I6I-172.

Blanche-Benveniste, C. et Jeanjean, C. 1988. Le français parlé: transcription et édition. Paris: Didier Érudition.

Bonvino, E., Masini, F. et Pietrandrea, P. 2009. List Constructions: A Semantic Network. In $3^{e}$ colloque international de l'Association française de linguistique cognitive AFLiCo 3: «Grammars in construction(s)》 (université Paris Ouest Nanterre La Défense, 27-29 mai 2009). I-20. En ligne à l'adresse suivante: http://francescamasini.caissa.it/ Presentations_files/parigi_draft.pdf. 
Bras, M., Prévot, L. et Vergez-Couret, M. 2008. Quelle(s) relation(s) de discours pour les structures énumératives? In J. Durand, B. Habert et B. LaKs (éd.), Actes $d u$ congrès mondial de Linguistique française - CMLF 2008 (Paris, 9-I2 juillet 2008). Paris : Institut de linguistique française: $1945^{-1964}$. En ligne à l'adresse suivante: https://www. linguistiquefrancaise.org/articles/cmlf/pdf/2008/or/cmlfo8225.pdf.

CADiot, P. 1988. De quoi ça parle? À propos de la référence de ça, pronom-sujet. Le français moderne $56(3-4):$ I74-189.

Corblin, F. 1995. Les formes de reprise dans le discours. Anaphores et chaînes de référence. Rennes: Presses universitaires de Rennes.

Corminboeuf, G. 2017. Comme ça, marqueur d'approximation. In F. Lefeuvre et G. Dostie (éd.), À l'articulation du lexique, de la grammaire et du discours: marqueurs grammaticaux et marqueurs discursifs. Paris: H. Champion: 263-282.

Dauzat, A. 1977. Les noms de famille de France. Traité d'anthroponymie française. Paris: Librairie Guénégaud [ $3^{\mathrm{e}}$ éd. revue et complétée par M.-T. Morlet].

Delafontaine, F. 2016. Machin, truc, chose dans les corpus oraux. Communication présentée lors des rencontres de Linguistique franco-suisses: «Corpus écrits, corpus oraux: de la morphosyntaxe au discours». Université de Neuchâtel, I3-I4 octobre 2016.

Dines, E. 1980. Variation in Discourse - “And Stuff like That”. Language in Society 9 (I): I3-3I.

Dubois, S. 1992. Extension Particles, etc. Language Variation and Change 4 (2): 179-203.

FERrÉ, G. 2009. Analyse multimodale des particules d'extension «et tout ça, etc.» en français. In H.-Y. Yoo et E. Delais-Roussarie (éd.), Actes de la conférence "Interface, discours et prosodie» - IDP 2009 (Paris, 9-II septembre 2009). I57-I7I. En ligne à l'adresse suivante : http://makino.linguist.jussieu.fr/idpog/docs/IDP_actes/Articles/Ferre.pdf.

FroneK, J. 1982. Thing as a Function Word. Linguistics 20 (9-10) : 633-654.

Fuchs, C. et Le Goffic, P. 2005. La polysémie de comme. In O. Soutet (éd.), La polysémie. Paris: Presses de l'université Paris-Sorbonne: 267-292.

GADET, F. (éd.) 20I7. Les parlers jeunes dans l'̂̂le-de-France multiculturelle. Paris: Ophrys.

Groupe De Fribourg 20I2. Grammaire de la période. Berne: P. Lang.

Halmøy, O. 2006. De chose et d'autres. La série truc, machin, bidule. Éléments de distribution. In M. Riegel, C. Schnedecker, P. Swiggers et I. Tamba (éd.), Aux carrefours du sens. Hommages offerts à Georges Kleiber pour son $60^{\circ}$ anniversaire. Paris - Louvain : Peeters : SII- 529 .

Johnsen, L. A. 20II. Un éclairage sur le fonctionnement référentiel de tout ça en fin de liste. In G. Corminboeuf et M.-J. BÉGuelin (éd.), Du système linguistique aux actions langagières. Mélanges en l'bonneur d'Alain Berrendonner. Bruxelles: De Boeck: 487-505.

Johnsen, L. A. 2017. L'expression de la sous-détermination dans les procédés de référence en français contemporain. Thèse de doctorat. Université de Neuchâtel.

Johnsen, L. A. et Avanzi, M. en prép. Examen des contours prosodiques de listes en français parlé.

Jucker, A. H., Smith, S. W. et Lüdge, T. 2003. Interactive Aspects of Vagueness in Conversation. Journal of Pragmatics 35 (12): 1737-1769. 
Kahane, S. et Pietrandrea, P. 20I2. La typologie des entassements en français. In F. Neveu, V. Muni Toke, P. Blumenthal, T. Klingler, P. Ligas, S. Prévost et S. Teston-Bonnard (éd.), SHS Web of Conferences. Actes du $3^{e}$ congrès mondial de Linguistique française - CMLF 2012 (Lyon, 4-7 juillet 20I2). Les Ulis: EDP Sciences. Vol. I : I809-1828. En ligne à l'adresse suivante: https://www.shs-conferences.org/ articles/shsconf/pdf/20I2/oI/shsconf_cmlfi2_ooo238.pdf.

KLEIBER, G. 1987. Mais à quoi sert donc le mot chose? Une situation paradoxale. Langue française 73 (I) : 109-I28.

MaIllard, M. 1989. Comment «ça» fonctionne ou étude du fonctionnement de «ça» en français moderne dans la perspective d'une linguistique génétique. Thèse de doctorat. Université Paris Io.

Minatsch, W. 2006. Machin, truc, chose: la naissance de marqueurs pragmatiques. In M. Drescher et B. Joв (éd.), Les marqueurs discursifs dans les langues romanes: approches théoriques et méthodologiques. Francfort-sur-le-Main : P. Lang: 153-172.

Muller, C. 20io. Les indéfinis dans les classes lexicales du français. Cabiers de lexicologie 96 (I) : 167-190.

Overstreet, M. 2005. And Stuff und so: Investigating Pragmatic Expressions in English and German. Journal of Pragmatics 37 (II): I845-I864.

Riegel, M., Pellat, J.-C. et Rioul, R. 2009. Grammaire méthodique du français. Paris: PUF [4 éd.].

Sales, M.-P. 2008. Influence du lexique et de la syntaxe sur la reprise pronominale: exemple $d e$ «ça». Thèse de doctorat. Université Paris Ouest Nanterre La Défense.

SCHNEDECKER, C. 1998. Les corrélats anaphoriques: une entrée en matière. In C. SCHNEDECKER (éd.), Les corrélats anaphoriques. Recherches linguistiques 22. Metz: université de Metz: 3-36.

Schnedecker, C. 20II. Monsieur Tout-le-monde, Maud Machin-Chouette, Denise Trucmuche et les autres... Inventaire et comportement des noms propres «indéfinis» du français. In D. Аmiot, W. De Mulder, E. Moline et D. Stosic (éd.), Ars Grammatica. Hommage à Nelly Flaux. Berne: P. Lang: 37-54.

Secova, M. 20I4. "Je sais et tout mais...” might the General Extenders in European French be Changing? Journal of French Language Studies 24 (2): 28I-304.

SPERber, D. et Wilson, D. 1989. La pertinence. Communication et cognition. Paris: Minuit.

Turco, G. et Coltier, D. I988. Des agents doubles de l'organisation textuelle: les marqueurs d'intégration linéaire. Pratiques 57: 57-79.

Willems, D. 1998. Un petit rien sur quelque chose. In A. Englebert, M. Pierrard, L. Rosier et D. VAn Raemdonck (éd.), La ligne claire. De la linguistique à la grammaire. Mélanges offerts à Marc Wilmet à l'occasion de son $60^{e}$ anniversaire. Paris - Bruxelles: Duculot: I37-I 45 .

\section{Corpus et dictionnaire}

ATILF (laboratoire Analyse et traitement informatique de la langue française). Base textuelle Frantext. En ligne à l'adresse suivante: http://www.frantext.fr/. 
ATILF (laboratoire Analyse et traitement informatique de la langue française). Trésor de la langue française informatisé (TLFi). En ligne à l'adresse suivante: http://atilf.atilf.fr/.

Avanzi, M., Béguelin, M.-J. et Diémoz, F. 20I2-20I5. Présentation du corpus OFROM - Corpus oral de français de Suisse romande. Université de Neuchâtel. En ligne à l'adresse suivante: http://www.unine.ch/ofrom.

Branca-Rosoff, S., Fleury, S., Lefeuvre, F. et Pires, M. (éd.) 2009. Discours sur la ville. Corpus de français parlé parisien des années 2000 (CFPP2000). Université Sorbonne Nouvelle - Paris 3. En ligne à l'adresse suivante: http://cfppzooo.univ-parisz.fr. 